www.nature.com/pj

\title{
Synthesis and memory performance of a conjugated polymer with an integrated fluorene, carbazole and oxadiazole backbone
}

\author{
Long-Jia Zeng ${ }^{1}$, Gang Liu $^{2}$, Bin Zhang ${ }^{1}$, Junneng Chen ${ }^{1}$, Yu Chen ${ }^{1,3}$ and En-Tang Kang ${ }^{2}$
}

A new soluble conjugated polymer, poly[\{9-(4-methoxyphenyl)-9H-carbazole\}(9,9-dioctylfluorene)(2,5-diphenyl-1,3,4-oxadiazole)] (PCFO), was synthesized through the Suzuki coupling reaction. The absolute fluorescence quantum yield of PCFO was measured using the integrating sphere of a photoluminescence spectrofluorometer, and changed from $49.1 \%$ for a dilute tetrahydrofuran solution to $16.2 \%$ for a thin film due to the existence of a strong fluorescence quenching effect in the solid state. The HOMOLUMO bandgap $(3.07 \mathrm{eV})$ calculated from the electrochemical measurement is nearly identical to the optical bandgap ( $3.06 \mathrm{eV})$ estimated from the ultraviolet/visible absorption onset data. This film was attached to aluminum and indium-tin oxide contacts to fabricate a memory device with typical bistable electrical switching, nonvolatile write-once read-many-times memory performance, a turn-on voltage of $\sim-2.3 \mathrm{~V}$ and an ON/OFF ratio of $\sim 10^{5}$. Degradation of the current density was observed for neither the ON nor OFF states after one hundred million continuous read cycles, which indicates that both states were insensitive to read cycles.

Polymer Journal (2012) 44, 257-263; doi:10.1038/pj.2011.114; published online 26 October 2011

Keywords: donor-acceptor system; molecular devices; polymer memory; synthesis

\section{INTRODUCTION}

Polymer memories are proposed to revolutionize electrical applications by providing extremely inexpensive, lightweight and transparent modules that can be fabricated onto plastic, glass or the top layer of CMOS hybrid integration circuits, ${ }^{1,2}$ and have been identified as an emerging memory technology by the International Technology Roadmap for Semiconductors since year 2005. ${ }^{3}$ Rather than encoding ' 0 ' and ' 1 ' as a charge stored in the cell of a silicon device, polymer memory stores data in an entirely different form, such as in a highand low-conductivity response to an applied voltage. ${ }^{4}$ The molecular structure of polymers can be tailored by functionalizing them with electron donors (D) and acceptors (A) of different strengths, spacer moieties of different steric effects for the electroactive pendant groups, or nanostructured electroactive materials, to induce different memory behaviors in simple metal/polymer/metal devices. ${ }^{5,6}$ Very recently, Liu and Chen ${ }^{7}$ highlighted the recent developments in D-A polymers for resistive switching memory device applications including conjugated polymers, functional polyimides, nonconjugated pendent polymers and polymer composites. D/A polymer materials (usually carbazole or fluorene donors) with moderate (or incomplete) charge transfer (CT) are likely to exhibit switching behaviors by external voltage bias. The strong dipole moment of molecule helps to sustain the conductive CT state and thus presents a nonvolatile behavior. The optimization of donor/acceptor ratios and the depth of trapping barrier define the volatile/nonvolatile nature. ${ }^{7}$

In our previous work, we designed a series of D-A-type functional polymers. ${ }^{8-19}$ The device based on conjugated poly[9,9-bis(4-diphenylaminophenyl)-2,7-fluorene] donors covalently bridged with Disperse Red 1 acceptors (DR1-PDPAF-DR1) exhibited an accessible rewritable memory characteristic. ${ }^{10,17}$ The excitation of donor promoted CT to the conjugated channel, finally the electrons were at LUMO1 or further at LUMO2 and gave rise to a conductive charge separation state. Polyfluorene-based copolymers containing the side-chain electron-rich TPA and electron-poor cyano substituents exhibited a typical write-once read-many-times (WORM) memory characteristic as the positive charges on the TPA or fluorene moieties were rapidly consumed by the cyano groups as a result of switching operation. ${ }^{14}$ TPA-based conjugated polyazomethine covalently grafted

\footnotetext{
1Shanghai Key Laboratory of Functional Materials Chemistry, Institute of Applied Chemistry, East China University of Science and Technology, Shanghai, PR China; ${ }^{2}$ Department of Chemical and Biomolecular Engineering, National University of Singapore, Kent Ridge, Singapore and ${ }^{3}$ The State Key Laboratory of ASIC \& System, Fudan University, Shanghai, China

Correspondence: Professor Y Chen, Shanghai Key Laboratory of Functional Materials Chemistry, Institute of Applied Chemistry, East China University of Science and Technology, 130 Meilong Road, Shanghai 200237, PR China.

E-mail: chentangyu@yahoo.com

or Professor E-T Kang, Department of Chemical and Biomolecular Engineering, National University of Singapore, 10 Kent Ridge 119260 , Singapore.

E-mail: cheket@nus.edu.sg

Received 1 August 2011; revised 7 September 2011; accepted 9 September 2011; published online 26 October 2011
} 
with graphene oxide (TPAPAM-GO) was directly used to fabricate nonvolatile rewritable memory devices. ${ }^{11}$ Electrons transmitted from the HOMO of the hole-transporting polymer TPAPAM into LUMO of graphene layer by intramolecular CT interaction established charge transport pathways and switched the device from the OFF to ON state. The effective electron delocalization in graphene nanosheets might stabilize the CT state of TPAPAM-GO, leading to a nonvolatile nature. The electrical bistable switching behavior of the pendent D-A polymer devices was affected by both the electronic structure and surface morphology. The electron-withdrawing DR1-functionalized PVK copolymers (PVDR) were fabricated for memory device as the active layer sandwiched between ITO and Al electrode. ${ }^{13}$ The PVDR film displayed helical columnar stacks with large grain sizes, whereas a non-aggregated PVDR film exhibited an amorphous morphology with a smaller grain size. Both the PVDR devices showed WORM performance with an ON/OFF current ratio of $10^{5}$. The asymmetric distribution of the HOMO and LUMO level could stabilize the conductive CT state of the PVDR copolymer leading to the nonvolatile and non-erasable behavior. Besides, the stability of the nano-aggregated PVDR device was much better than that of the non-aggregated PVDR device. The surface defects and large numbers of the grain boundaries in the amorphous PVDR sample acted as charge-trapping centers. An effective charge transport channel and better polymer/ metal contacts of the self-assembled smooth PVDR film contributed to the more stable ON state current in the corresponding device. Similar WORM devices were constructed from two PVK derivatives with the pendent azobenzene chromopheres and terminal electron acceptor moieties $\left(-\mathrm{NO}_{2}\right.$ or $\left.-\mathrm{CN}\right) .{ }^{15}$ Electric field-formed high dipole moments originally from increased charge separation upon CT led to the nonvolatile behavior.

For polymer memory materials, it is very important and profitable to achieve a more balanced charge transport by integrating both electron acceptors and donors into the polymer. The electron-deficient 2,5-diphenyl-1,3,4-oxadiazole (OXD) and its derivatives have been recognized as excellent electron acceptors because of their good chemical, thermal and optical stabilities, as well as the high fluorescence quantum yields. ${ }^{20}$ Usually, the OXD units are covalently bonded to emissive polymers or small molecules to enhance the electron injection and transport in the emission layer. ${ }^{21-27}$ For example, Jen et al. $^{26}$ designed a fluorene-based alternating copolymer, PF-OXD, which contained OXD moieties with their phenyl-end group directly attached to the C-9 carbon in every other fluorene unit. The improved device performance over poly(9,9-dioctylfluorene) is due to the better electron injection and transport in PF-OXD, and the efficient energy transfer from the OXD side chain to the PF main chains. By using electron-donating 9-(4-vinylphenyl)carbazole and electron-accepting 2-phenyl-5-(4-vinylphenyl)-1,3,4-oxadiazole or 2-(4-vinylbiphenyl)-5(4-phenyl)-1,3, 4-oxadiazole as side chains, Chen and colleagues ${ }^{28}$ prepared a nonconjugated random copolymer by the nitroxidemediated free-radical polymerization method. Both experimental and theoretical results indicated that the CT between the pendant donor and acceptor was relatively weak without significant orbital hybridization. Ling et al. ${ }^{29}$ synthesized a nonconjugated methacrylate copolymer ( $\mathrm{PCzOxEu}$ ) that contained carbazole, OXD and europium complexes in the pendant groups by free-radical copolymerization of methacrylate monomers containing the respective functional groups. The large dipole moment of OXD in PCzOxEu favors the separation of charges, which accounts for the longer retention time and improved stability of the present memory device.

In this contribution, we designed and synthesized a new conjugated copolymer containing an electron-accepting $\mathrm{OXD}$, electron-donating carbazol and dioctylfluorene units in the polymer main chain, poly [ $\{9-$ (4-methoxyphenyl)-9H-carbazole\}(9,9-dioctylfluorene) (2,5-diphenyl1,3,4-oxadiazole)](PCFO, Scheme 1). This polymer exhibited WORM memory effects in an Al/polymer/ITO structure with a switching threshold voltage of $-2.3 \mathrm{~V}$ and an ON/OFF current ratio of $\sim 10^{5}$. Basically, WORM memory, a type of nonvolatile memory capable of permanently holding data and being repeatedly read, is desirable for the ultra low cost and permanent storage of digital images, and eliminates the need for the slow, bulky and expensive mechanical drives used in conventional magnetic and optical memories. ${ }^{13-15,30-38}$

\section{EXPERIMENTAL PROCEDURE}

\section{General}

All of the synthetic steps before the termination reaction were carried out under purified argon, and all chemicals were purchased from Aldrich (Shanghai, China) and used without further purification. Organic solvents were purified, dried and distilled under dry nitrogen. The preparation of 2,5-bis(4-bromophenyl)-1,3,4-oxadiazole resulted in a $70 \%$ yield, according to the method reported in the literature..$^{21}$

Weight-average $(M w)$ and number-average molecular weights $(\mathrm{Mn})$ were determined by gel permeation chromatography (GPC). Four Waters (Ultrastyragel, Milford, MA, USA) columns $\left(300 \times 7.7 \mathrm{~mm}^{2}\right.$, guarded, $10^{5}, 10^{4}, 10^{3}$ and $5 \times 10^{2} \AA$ pore size gel in series) were used for the GPC measurements with tetrahydrofuran (THF, $1 \mathrm{ml} \mathrm{min}^{-1}$ ) as the eluent. A monodispersed polystyrene sample was used as the molecular weight standard. Elemental microanalysis (for $\mathrm{C}, \mathrm{H}$ and $\mathrm{N}$ ) was performed on a Perkin-Elmer 2400 elemental analyzer (PerkinElmer, Waltham, MA, USA). ${ }^{1} \mathrm{H}$ NMR spectra were measured on a Bruker Advance 500 NMR spectrometer (Bruker, Ettlingen, Germany) with $\mathrm{CDCl}_{3}$ as the solvent and tetramethylsilane as the internal standard. Thermogravimetric analysis (TGA) was conducted on a TGA 2050 thermogravimetric analyzer (Perkin-Elmer) using a heating rate of $10{ }^{\circ} \mathrm{C} \mathrm{min}{ }^{-1}$ and a nitrogen flow rate of $40 \mathrm{ml} \mathrm{min}{ }^{-1}$. Cyclic voltammetry measurements were performed with an Autolab potentiostat/galvanostat system (Autolab, Utrecht, Netherlands) using a three-electrode cell under an argon atmosphere. The polymer film was coated onto a Pt disk electrode (working electrode) and scanned anodically and cathodically in a $\mathrm{Bu}_{4} \mathrm{NClO}_{4}$ solution in dry acetonitrile $(0.1 \mathrm{M})$ with an $\mathrm{Ag}$ / $\mathrm{AgCl}$ and platinum wire as the reference and counter electrode, respectively.

\section{Device fabrication and characterization}

The electrical properties of PCFO were evaluated using an Al/polymer/ITO sandwich structure. The ITO-glass substrates were pre-cleaned in an ultrasonic bath for $15 \mathrm{~min}$ each in detergent, deionized water, acetone and isopropyl alcohol, and a $50 \mu$ polymer solution in toluene was spin coated onto the precleaned ITO substrate. The resultant film thickness is $\sim 100 \mathrm{~nm}$ as measured by a step profiler. After drying the film under reduced pressure $\left(10^{-5}\right.$ Torr $)$ at room temperature overnight, the $\mathrm{Al}$ top electrodes were deposited onto the film surface via thermal evaporation at $10^{-7}$ Torr through a shadow mask. The top electrodes are $0.4 \times 0.4 \mathrm{~mm}^{2}$ in area and $\sim 300 \mathrm{~nm}$ in thickness. The devices were characterized under ambient conditions, using a Hewlett-Packard 4155B semiconductor parameter analyzer (Hewlell-Packard, Palo Alto, CA, USA) with an Agilent 16440A SMU/pulse generator (Agilent, Santa Clara, CA, USA).

\section{Synthesis of 9-(4-methoxyphenyl)-9H-carbazole}

A mixture of 1-iodo-4- methoxybenzene $(5.5 \mathrm{~g}, 24 \mathrm{mmol})$, carbazole $(3.34 \mathrm{~g}$, $20 \mathrm{mmol}), \mathrm{K}_{2} \mathrm{CO}_{3}(2.77 \mathrm{~g}, 20 \mathrm{mmol}), 1,10$-phenanthridine $(0.72 \mathrm{~g}, 4 \mathrm{mmol})$ and CuI $(0.76 \mathrm{~g}, 4 \mathrm{mmol})$ in dry $\mathrm{N}, \mathrm{N}$-dimethylformamide (DMF) $(50 \mathrm{ml})$ was heated at $155^{\circ} \mathrm{C}$ for $24 \mathrm{~h}$. After cooling to room temperature, a larger volume of ice water was poured into the reaction mixture and the resultant precipitate was collected by vacuum filtration. The collected solid was washed with distilled water and anhydrous methanol, and the crude product was further purified by column chromatography using silica gel with petroleum ether/ethyl acetate (v/v 5:1) as the eluent to afford a white solid. Yield: $4.5 \mathrm{~g}(82 \%) .{ }^{1} \mathrm{H}$ $\operatorname{NMR}\left(\mathrm{CDCl}_{3}, 500 \mathrm{MHz}\right): \delta$ (p.p.m.) $=8.14(\mathrm{~d}, J=7.7 \mathrm{~Hz}, 2 \mathrm{H}), 7.46-7.44(\mathrm{~m}$, $2 \mathrm{H}), 7.42-7.38(\mathrm{~m}, 2 \mathrm{H}), 7.32(\mathrm{~d}, J=8.1 \mathrm{~Hz}, 2 \mathrm{H}), 7.28-7.24(\mathrm{~m}, 2 \mathrm{H}), 7.12-7.10$ $(\mathrm{m}, 2 \mathrm{H}), 3.92(\mathrm{~s}, 3 \mathrm{H})$; electron affinity (EA) calculated for $\mathrm{C}_{19} \mathrm{H}_{15} \mathrm{NO}$ 

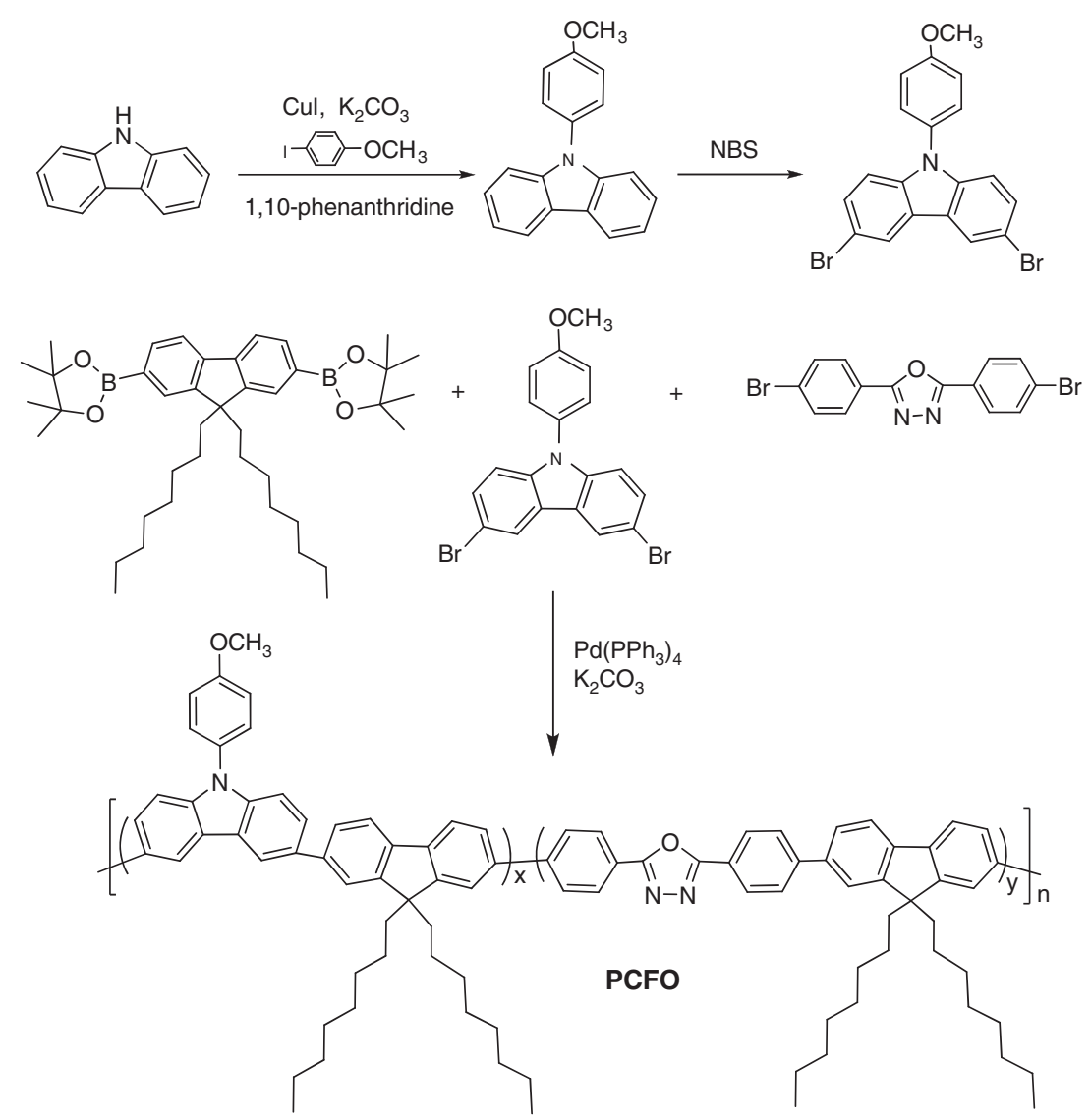

Scheme 1 Synthesis of PCFO.

(273.12 $\left.\mathrm{g} \mathrm{mol}^{-1}\right)$ : C $83.49, \mathrm{H} 5.53, \mathrm{~N} \mathrm{5.12;} \mathrm{found:} \mathrm{C} 83.35, \mathrm{H} 5.24, \mathrm{~N} 5.13$; EI-MS: $m / z 273.1\left(\mathrm{M}^{+}\right)$.

\section{Synthesis of 3,6-dibromo-9-(4-methoxyphenyl)-9H-carbazole} 9-(4-methoxyphenyl)-9H-carbazole $(0.74 \mathrm{~g}, 2.7 \mathrm{mmol})$ in dry DMF $(50 \mathrm{ml})$ was cooled to $0^{\circ} \mathrm{C}$, and $\mathrm{N}$-bromosuccinimide $(1.07 \mathrm{~g}, 6.0 \mathrm{mmol})$ was added dropwise over $1.5 \mathrm{~h}$ with magnetic stirring. The temperature was allowed to rise to room temperature while stirring was maintained for a total of $4 \mathrm{~h}$. Ice water was then poured into the reaction mixture to give a white precipitate. Evaporation of the solvent was followed by recrystallization from toluene/n-hexane to afford a white solid with a $90 \%$ yield $(1.05 \mathrm{~g}) .{ }^{1} \mathrm{H}-\mathrm{NMR}\left(\mathrm{CDCl}_{3}, 500 \mathrm{MHz}\right): \delta$ (p.p.m.) $=8.19(\mathrm{~m}, 2 \mathrm{H}), 7.50-7.47(\mathrm{~m}, 2 \mathrm{H}), 7.40-7.37$ (m, 2H), 7.18-7.10 $(\mathrm{m}, 4 \mathrm{H}), 3.92(\mathrm{~s}, 3 \mathrm{H})$. EA calculated for $\mathrm{C}_{19} \mathrm{H}_{13} \mathrm{Br}_{2} \mathrm{NO}\left(431.12 \mathrm{~g} \mathrm{~mol}^{-1}\right)$ : C52.93, H3.04, N3.25; found C 52.25, H 3.07, N 3.23; EI-MS: $m / z 431.0\left(\mathrm{M}^{+}\right)$.

\section{Synthesis of the polymer PCFO}

To a 50-ml Schlenk tube charged with 3,6-dibromo-9-(4-methoxyphenyl)9H-carbazole $(227 \mathrm{mg}, \quad 0.53 \mathrm{mmol}), \quad 2,5$-bis(4-bromophenyl)-1,3,4-oxadiazole (50 mg, $\quad 0.13 \mathrm{mmol}), \quad 2,2^{\prime}$-(9,9-dioctyl-9H-fluorene-2,7-diyl)bis(4,4,5,5-tetramethyl-1,3,2-dioxaborolane) $(423 \mathrm{mg}, 0.66 \mathrm{mmol})$ and potassium carbonate $(552.2 \mathrm{mg}, 4.00 \mathrm{mmol})$ were added together with $\mathrm{Pd}\left(\mathrm{PPh}_{3}\right)_{4}(2.5 \mathrm{mg}$, $0.002 \mathrm{mmol})$ in a glove box. Degassed toluene $(10 \mathrm{ml})$ and water $(2 \mathrm{ml})$ were added into the mixture by syringe. After heating the mixture at $85^{\circ} \mathrm{C}$ under an argon atmosphere for $36 \mathrm{~h}$, the crude product was extracted three times with chloroform, and the combined organic layers were washed with water and brine, dried over anhydrous sodium sulfate and filtered. The obtained filtrate was concentrated into a small volume, and then added dropwise into stirred methanol. After filtration, the collected solid was purified by reprecipitating into methanol, and then performing a Soxhlet extraction with acetone. The polymer was dried under vacuum to give $460 \mathrm{mg}$ of a light yellow solid with a yield of $65 \%$. GPC (THF): $M_{\mathrm{n}}=6.33 \times 10^{3}, M_{\mathrm{w}}=1.05 \times 10^{4}$, $\mathrm{PDI}=1.66 ;{ }^{1} \mathrm{H}-\mathrm{NMR}\left(\mathrm{CDCl}_{3}, 500 \mathrm{MHz}\right): \delta$ (p.p.m. $)=8.53-8.28(\mathrm{~m}, 3 \mathrm{H}), 7.86-$ $7.38(\mathrm{~m}, 15 \mathrm{H}), 7.19-7.00(\mathrm{~m}, 2 \mathrm{H}), 3.96(\mathrm{~s}, 3 \mathrm{H}), 2.10(\mathrm{~m}, 5 \mathrm{H}), 1.11$ $(\mathrm{m}, 24 \mathrm{H}), 0.80-0.76(\mathrm{~m}, 14 \mathrm{H})$.

\section{RESULTS AND DISCUSSION}

PCFO was synthesized through a Suzuki coupling reaction between the monomers 3,6-dibromo-9-(4-methoxyphenyl)-9H-carbazole, 2, 5-bis(4-bromophenyl)-1,3,4-oxa- diazole and 2,2'-(9,9-dioctyl-9Hfluorene-2,7-diyl)bis(4,4,5,5-tetramethyl-1,3,2-dioxaboro-lane) in the presence of tetrakis(triphenyl-phosphine)palladium $(0)$ as the catalyst. This copolymer has good solubilities in several common organic solvents such as THF, toluene, dichloromethane, chloroform and chlorobenzene, and was found to have an $M_{\mathrm{n}}$ of $6.33 \times 10^{3}$ with a polydispersity of 1.66 by GPC analysis against a linear polystyrene standard.

The thermal properties of PCFO were measured by TGA under a nitrogen atmosphere. As shown in Figure 1, the TGA thermogram exhibits two weight loss plateaus. At lower temperatures, almost no weight loss was observed. The copolymer first decomposed at $310-500^{\circ} \mathrm{C}$ (the onset temperature for the thermal bond cleavage was $425^{\circ} \mathrm{C}$ ), and then was subjected to a second decomposition at $510-750{ }^{\circ} \mathrm{C}$ (the onset temperature for the thermal bond cleavage was $604^{\circ} \mathrm{C}$ ). The weight loss at the first plateau is $38 \%$, which is in good agreement with the content (38.1\%) of methoxyl and octyl side chains in the polymer.

As shown in Figure 2, the main absorption peaks in the ultraviolet/ visible (UV/Vis) absorption spectrum of PCFO are located at 


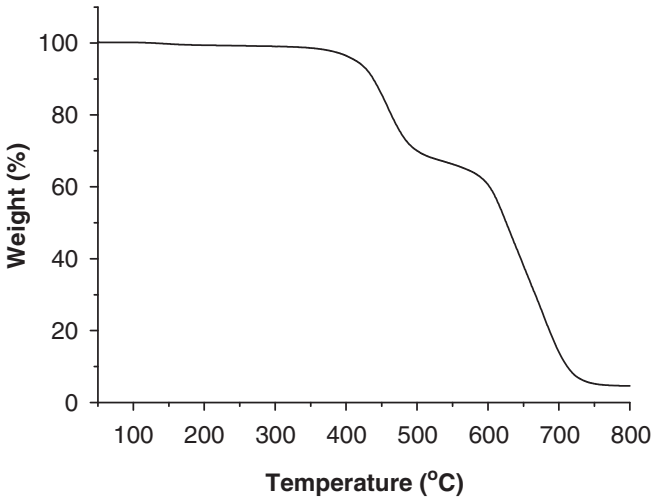

Figure 1 TGA thermogram of the PCFO copolymer measured under a nitrogen atmosphere.

315 (shoulder) and $353 \mathrm{~nm}$ due to the $\pi-\pi^{\star}$ transition in the polymer backbone. Compared with the dilute solution, the absorption spectrum of the PCFO thin film red shifted by $\Delta \lambda=2 \mathrm{~nm}$, and broadened to include a wing beyond $700 \mathrm{~nm}$. Upon excitation at $355 \mathrm{~nm}$ by a continuous laser (linear optical regime), PCFO gains a strong blue light emission peak centered at $444 \mathrm{~nm}$ for the dilute THF solution and $445 \mathrm{~nm}$ for the thin film. The absolute fluorescence quantum yield $\left(\Phi_{\mathrm{f}}\right)$ of PCFO was measured using the integrating sphere of a photoluminescence spectrofluorometer and changed from $49.1 \%$ for dilute THF solution to $16.2 \%$ for a thin film due to the existence of a strong fluorescence quenching effect in the solid state. The larger Stokes shift observed for PCFO $\left(5646 \mathrm{~cm}^{-1}\right.$ in THF; $5539 \mathrm{~cm}^{-1}$ in the solid state) is related to the energy difference between the $0-0$ transitions in the photoluminescence and absorption spectra. The relaxation of either the electronic or geometrical structure of the photoexcited molecule, in general, generates a large Stokes shift. ${ }^{39}$

The cyclic voltammetry curve of the PCFO film coated onto Pt is shown in Figure 3. The potentials were measured against an $\mathrm{Ag} / \mathrm{AgCl}$ reference electrode and converted to values versus the SCE by adding $0.29 \mathrm{~V}^{40}$ The first oxidation and reduction potentials for PCFO were found to be $+1.42 \mathrm{~V}$ versus $\mathrm{Ag} / \mathrm{Ag}^{+}$, which corresponds to $+1.71 \mathrm{~V}$ versus $\mathrm{SCE}$, and $-1.65 \mathrm{~V}$ versus $\mathrm{Ag} / \mathrm{Ag}^{+}$, which corresponds to $-1.36 \mathrm{~V}$ versus $\mathrm{SCE}$, respectively. The calculated $\mathrm{HOMO}$ and LUMO values were -5.84 and $-2.77 \mathrm{eV}$, respectively, and the HOMO-LUMO bandgap calculated from the electrochemical measurement was $3.07 \mathrm{eV}$, which is in good agreement with that estimated from the UV/Vis absorption onset data obtained for the dilute solution $\left(E_{\mathrm{g}}^{\text {opt }}=1243.37 / \lambda_{\text {onset }}=1243.37 / 406=3.06 \mathrm{eV}\right)$. The values of the ionization potential (IP) and EA can also be estimated from these onset potentials (versus SCE) using equations (1) (2). ${ }^{41-45}$

$$
\mathrm{IP}=E_{\text {first oxidation }}+4.39 \mathrm{eV}
$$

$$
\mathrm{EA}=E_{\text {first reduction }}+4.39 \mathrm{eV}
$$

where the constant $4.39 \mathrm{eV}$ is the relationship between the IP, EA and the electrochemcial potentials. ${ }^{46}$ The calculated IP and EA values are 6.10 and $3.03 \mathrm{eV}$, respectively.

The electrical conductivity switching and memory effects of the $\mathrm{Al} /$ polymer/ITO sandwich structure devices are illustrated by the graph of current density versus voltage (Figure 4a). Starting with the low-conductivity state in the as-fabricated device, which is referred to as the OFF state, the current density of the Al/PCFO/ITO device increases slowly with increasing applied voltage but remains low
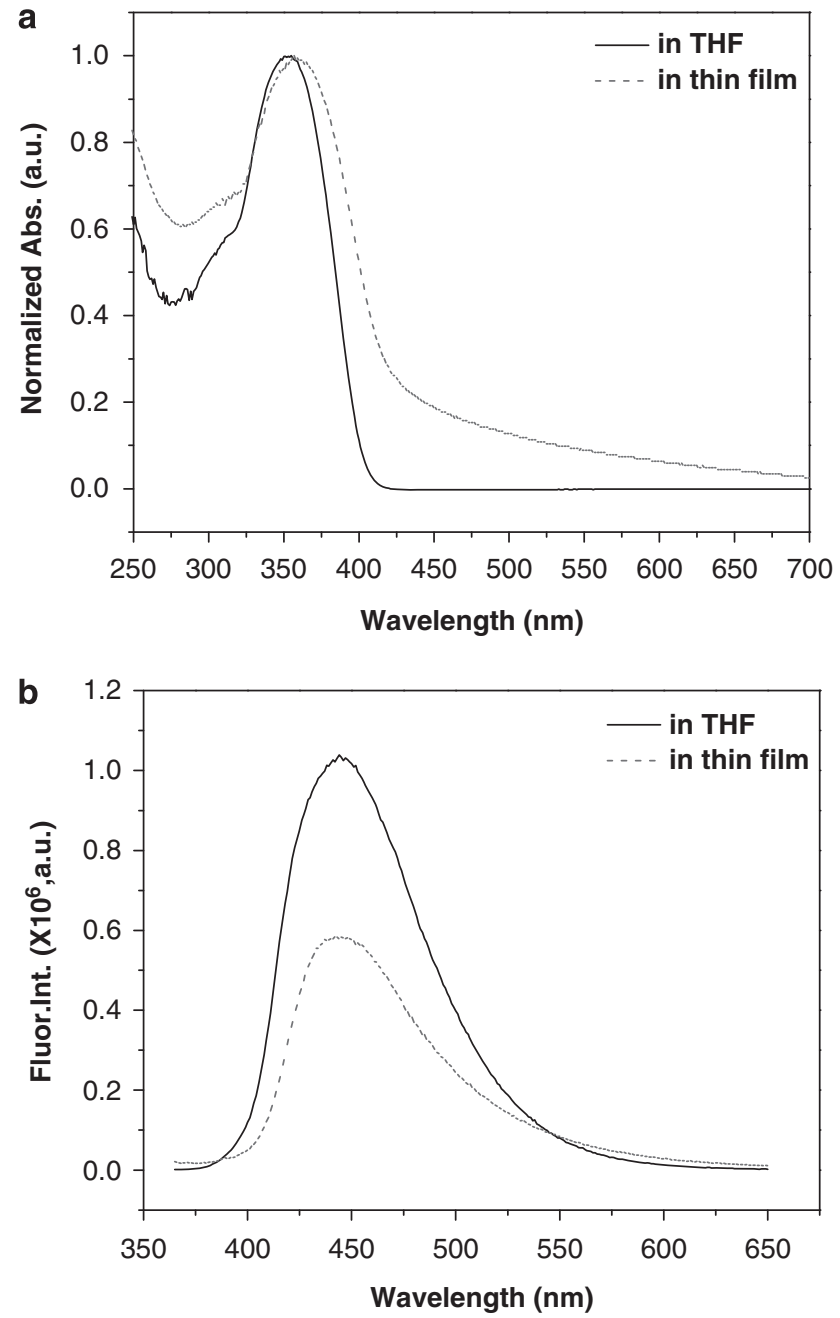

Figure 2 UV/Vis absorption (a) and photoluminescence (b) $\left(\lambda_{\mathrm{ex}}=355 \mathrm{~nm}\right)$ spectra of PCFO in a dilute THF solution and as a thin film. A full color version of this figure is available at Polymer Journal online.

$\left(\sim 10^{-7}-10^{-8} \mathrm{Acm}^{-2}\right)$ until the threshold voltage of $-2.3 \mathrm{~V}$ is reached. At the threshold voltage, the current density abruptly increases, and the device transitions from the low-conductivity state (the OFF state) to the high-conductivity state (the ON state) with an ON/OFF current ratio of $\sim 10^{5}$ (from $\sim 10^{-7}$ to $\sim 10^{-2} \mathrm{Acm}^{-2}$ ), which is high enough to promise a low misreading rate through the precise control of the ON and OFF states. The ON state device retains its high-conductivity state after repeated forward and reverse biased sweeps with applied voltages of up to $\pm 3 \mathrm{~V}$ as well as when the power supply is turned off, which demonstrates the Al/PCFO/ITO sandwich device nonvolatile WORM electronic memory characteristics. No significant degradation of either the ON or OFF states in the device was observed after $1 \times 10^{4} \mathrm{~s}$ of the continuous stress test (Figure $4 \mathrm{~b}$ ), which indicates that both the material and the electrode/polymer interfaces are stable. The current density of the ON and OFF states (especially the ON state) remains constant even after one hundred million $\left(10^{8}\right)$ read cycles, which indicates both states (especially the $\mathrm{ON}$ state) were insensitive to the read cycles (Figure 4c). Upon proper encapsulation, the performance of the $\mathrm{Al} /$ polymer/ITO electronic memory devices is expected to improve.

With the presence of both an electron donor (carbazole and fluorene moieties) and acceptor (OXD) in the PCFO polymer, it is 


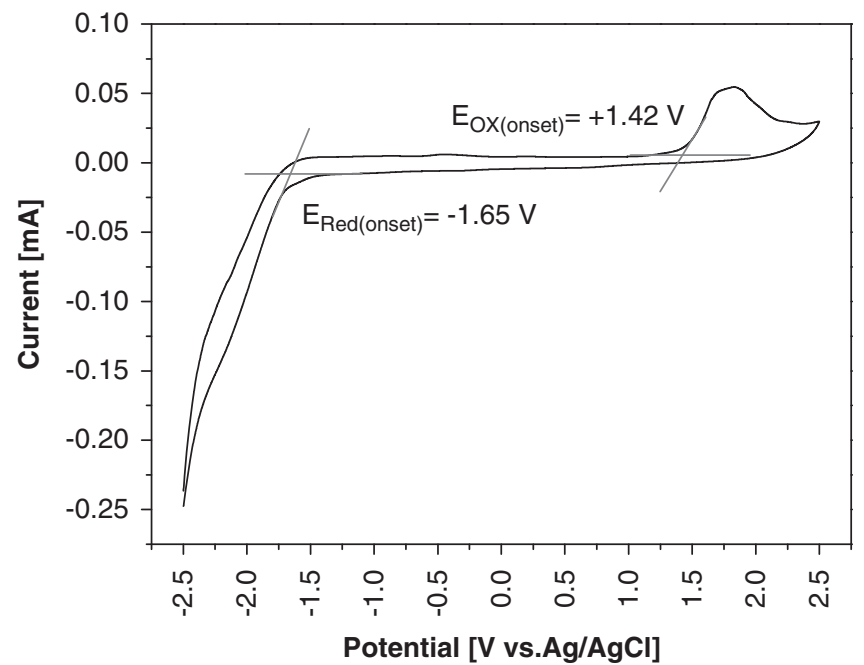

Figure 3 Cyclic voltammogram of the PCFO film on a Pt electrode with $\mathrm{Bu}_{4} \mathrm{NClO}_{4}(0.1 \mathrm{M})$ as the supporting electrolyte. Sweep rate $100 \mathrm{mVs}^{-1}$. A full color version of this figure is available at Polymer Journal online.

reasonable to expect that the intramolecular CT interactions might be responsible for the electrical conductivity switching and memory effects in the resultant polymer. Because of the energy difference between the HOMO and LUMO of the polymer, and the work function of the metal electrodes, Schottky barriers are formed at both the Al/polymer and polymer/ITO interfaces during the negative voltage sweeps. The lowest energy barrier between the work function of ITO $(-4.8 \mathrm{eV})$ and the HOMO level $(-5.84 \mathrm{eV})$ of PCFO is $1.04 \mathrm{eV}$, which suggests that hole injection from ITO (as the anode) into the HOMO of PCFO (corresponding to a negatively bias) is favored. The electron injection from $\mathrm{Al}(-4.3 \mathrm{eV})$ into the LUMO $(-2.77 \mathrm{eV})$ of PCFO will be relatively difficult as a result of the higher energy barrier $(1.53 \mathrm{eV})$. These findings indicated that PCFO can serve as a p-type (hole-transporting) material and that holes dominate the conduct process. ${ }^{47,48}$ It is known that, depending on the nature of the charge carriers, the semiconductor can function as either $\mathrm{p}$ - or n-type semiconductors. In p-type semiconductors, the majority of the carriers are holes with good mobility, whereas in n-type semiconductors, the majority of the carriers are electrons. During the negative sweep, electrons are injected from the Al top electrode while holes are injected from the ITO bottom electrode into the polymer thin film. The incorporated electron-accepting OXD moieties could serve as the hole-blocking layer in the active memory layer. ${ }^{28}$ Under a low electrical field, the trapped charge carriers will induce a charged layer in the polymer film near the electrodes and prevent further charge injection from the electrodes. Under a high electric field with sufficient energy to overcome the charge-trapping barrier, intramolecular CTs can occur between the electron donor and acceptor. The resultant CT complex in the D-A structure is more conductive than the pristine polymer film. At this stage, the majority of the charge traps are filled, and a 'trap-free' environment with higher charge carrier mobilities is formed throughout the entire polymer film. Thus, the polymer device transitions from the low-conductivity state (OFF state) to the high-conductivity state (ON state).

\section{CONCLUSION}

A new conjugated polymer, PCFO, was synthesized through a Suzuki coupling reaction using tetrakis-(triphenylphosphine)palladium $(0)$ as the catalyst. This copolymer exhibited good solubilities in several
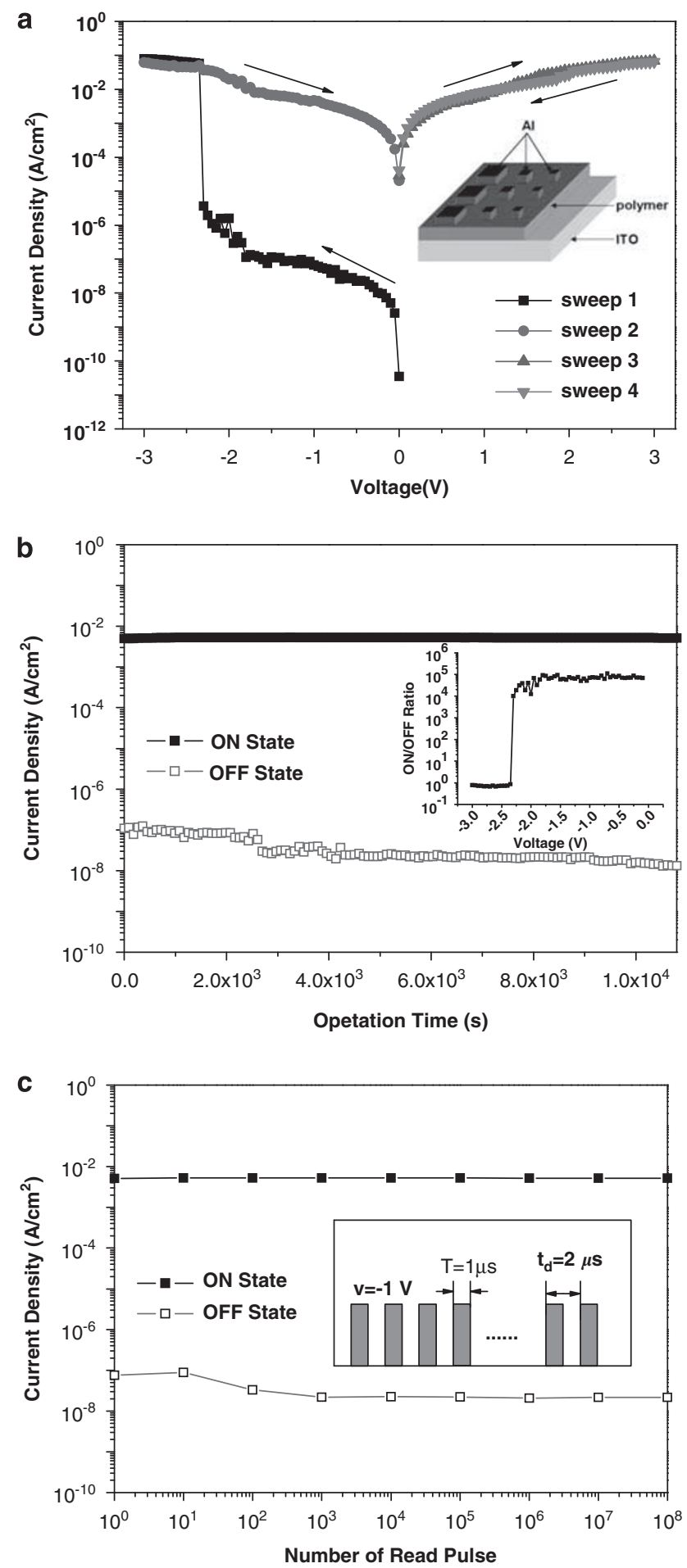

Figure 4 (a) Typical $I-V$ characteristics of the WORM memory device in both the ON and OFF states. The inset shows the device structure; (b) the effect of operation time (at $-1 \mathrm{~V}$ ) on the device current density in both the OFF and $\mathrm{ON}$ states tested under ambient conditions. The inset shows the ONto OFF-state ratio as a function of the applied voltage for the same sweep; (c) the effect of the $-1 \mathrm{~V}$ read pulses on both the ON and OFF state currents in the ITO/PCFO/Al device. The inset shows the pulse used for measurement. A full color version of this figure is available at Polymer Journal online. 
common organic solvents and good thermal stability. The absolute fluorescence quantum yield of PCFO changed from $49.1 \%$ for a dilute THF solution to $16.2 \%$ for a thin film due to the existence of a strong fluorescence quenching effect in the solid state. The HOMO, LUMO, IP and EA values experimentally estimated from the onset of the redox potentials were $-5.84,-2.77,6.10$ and $3.03 \mathrm{eV}$, respectively. The HOMO-LUMO bandgap $(3.07 \mathrm{eV})$ calculated from the electrochemical measurement is nearly identical to the optical bandgap $(3.06 \mathrm{eV})$ estimated from the UV/Vis absorption onset data. A WORM memory device was fabricated by spin coating a PCFO solution as the active layer sandwiched between aluminum electrode and an indium-tin oxide electrodes. This device showed very good memory performance, with an ON/OFF current ratio of $\sim 10^{5}$, which is high enough to provide a low misreading rate through the precise control of the $\mathrm{ON}$ and OFF states. Intramolecular CT might be responsible for the electrical conductivity switching and memory effects of this polymer.

\section{ACKNOWLEDGEMENTS}

We are grateful for the financial support of the National Natural Science Foundation of China (21074034), the Ministry of Education of China (309013), the Fundamental Research Funds for the Central Universities, the State Key Laboratory of ASIC \& System of Fudan University (11KF007), the Shanghai Municipal Educational Commission for the Shuguang fellowship (08GG10) and the Shanghai Eastern Scholarship.

1 Kuang, Y., Huang, R., Tang, Y., Ding, W., Zhang, L. \& Wang, Y. Flexible singlecomponent-polymer resistive memory for ultrafast and highly compatible nonvolatile memory applications. IEEE. Electr. Device Lett. 31, 758-760 (2010).

2 Song, Y., Ling, Q. D., Zhu, C., Kang, E. T., Chan, D. S. H., Wang, Y. H. D. \& Kwong, L. Memory performance of a thin-film device based on a conjugated copolymer containing fluorene and chelated europium complex. IEEE Electr. Device Lett. 27, 154-156 (2006).

3 Emerging research devices. In: International Technology Roadmap for Semiconductors (ITRS) 2005 edn 1-70 (Semiconductor Industry Association, International Sematech, Austin, TX, 2005).

4 Ling, Q. D., Zhu, C. X., Chan, D. S. H., Kang, E. T. \& Neoh, K. G. in Encyclopedia of Nanoscience and Nanotechnology 2nd edn (ed. Nalwa, H.S.) (American Scientific Publishers, Stevenson Ranch, CA, USA, 2007).

5 Ling, Q. D., Liaw, D. J., Teo, E. Y. H., Zhu, C. X., Chan, D. S. H., Kang, E. T. \& Neoh, K. G. Polymer memories: bistable electrical switching and device performance. Polymer 48, 5182-5201 (2007).

6 Ling, Q. D., Liaw, D. J., Zhu, C., Chan, D. S. H., Kang, E. T. \& Neoh, K. G. Polymer electronic memories: materials, devices and mechanisms. Prog. Polym. Sci. 33, 917-978 (2008).

7 Liu, C. L. \& Chen, W. C. Donor-acceptor polymers for advanced memory device applications. Polym. Chem. 2, 2169-2174 (2011).

8 Lin, Y., El-Khouly, M. E., Chen, Y., Supur, M., Gu, L., Li, Y. \& Fukuzumi, S. A New cyanofluorene-triphenylamine copolymer: synthesis and photoinduced intramolecular electron transfer processes. Chem. Eur. J. 15, 10818-10824 (2009).

9 Chen, Y., El-Khouly, M. E., Zhuang, X. D., He, N., Araki, Y., Lin, Y. \& Ito, O. Synthesis and photoinduced electron-transfer process of a novel triphenylamine-substituted polyfluorene-C60 triad. Chem. Eur. J. 13, 1709-1714 (2007).

10 El-Khouly, M. E., Chen, Y., Zhuang, X. D. \& Fukuzumi, S. Long-lived charge-separated configuration of a push-pull archetype of disperse red 1 end-capped poly[9,9-bis (4-diphenylaminophenyl)fluorene]. J. Am. Chem. Soc. 131, 6370-6371 (2009).

11 Zhuang, X. D., Chen, Y., Liu, G., Li, P. P., Zhu, C. X., Kang, E. T., Noeh, K. G., Zhang, B., Zhu, J. H. \& Li, Y X. Conjugated-polymer-functionalized graphene oxide: synthesis and nonvolatile rewritable memory effect. Adv. Mater. 22, 1731-1735 (2010).

12 Liu, G., Zhuang, X. D., Chen, Y., Zhang, B., Zhu, J. H., Zhu, C. X., Neoh, K. G. \& Kang, E. T. Bistable electrical switching and electronic memory effect in a solutionprocessable graphene oxide-donor polymer complex. Appl. Phys. Lett. 95, 253301/ $1-253301 / 3$ (2009).

13 Zhuang, X. D., Chen, Y., Liu, G., Zhang, B., Neoh, K. G., Kang, E. T., Zhu, C. X. \& Li, Y. $X$. Preparation and memory performance of a nanoaggregated dispersed red 1-functionalized poly( $\mathrm{N}$-vinylcarbazole) film via solution-phase self-assembly. Adv. Funct. Mater. 20, 2916-2922 (2010).

14 Zhuang, X. D., Chen, Y., Li, B. X., Ma, D. G., Zhang, B., Li, Y. X. \& He, N. Polyfluorenebased push-pull type functional materials for write- once- read- many -times memory devices. Chem. Mater. 22, 4455-4461 (2010).
15 Liu, G., Zhang, B., Chen, Y., Zhu, C. X., Zeng, L. J., Chan, D. S. H., Neoh, K. G. \& Kang, E. T. Electrical conductivity switching and memory effects in poly(N-vinylcarbazole) derivatives with pendant azobenzene chromophores and terminal electron acceptor moieties. J. Mater. Chem. 21, 6027-6033 (2011).

16 Zhang, B., Liu, Y. L., Chen, Y., Neoh, K. G., Li, Y. X., Zhu, C. X., Tok, E. S. \& Kang, E. T. Nonvolatile rewritable memory effects in graphene oxide functionalized by conjugated polymer containing fluorene and carbazole units. Chem. Eur. J. 17, 10304-10311 (2011).

17 Ling, Q. D., Kang, E. T., Neoh, K. G., Chen, Y., Zhuang, X. D., Zhu, C. \& Chan, D. S. H. Thermally stable polymer memory devices based on a $\partial$-conjugated triad. Appl. Phys. Lett. 92, 143302/1-143302/3 (2008).

18 Zhang, B., Chen, Y., Zhuang, X. D., Liu, G., Yu, B., Kang, E. T. \& Li, Y X. Poly (N-vinylcarbazole) chemically modified graphene oxide. J. Polym. Sci. A: Polym. Chem. 48, 2642-2649 (2010).

19 Zhang, B., Chen, Y., Xu, L. Q., Zeng, L.J., He, Y., Kang, E.T. \& Zhang, J.J. Growing poly(N-vinylcarbazole) from the surface of graphene oxide via RAFT polymerization. J. Polym. Sci. A: Polym. Chem. 49, 2043-2050 (2011).

20 Schultz, B., Bruma, M. \& Brehmer, L. Aromatic poly(1,3,4-oxadiazoe)s as advanced materials. Adv. Mater. 9, 601-613 (1997).

21 Zhan, X. W., Liu, Y. Q., Wu, X., Wang, S. \& Zhu, D. B. New series of blue-emitting and electron-transporting copolymers based on fluorene. Macromolecules 35, 2529-2537 (2002).

22 Ding, J. F., Day, M., Robertson, G. \& Roovers, J. Synthesis and characterization of alternating copolymers of fluorene and oxadiazole. Macromolecules 35, 3474-3483 (2002).

23 Oyston, S., Wang, C. S., Hughes, G., Batsanov, A. S., Perepichka, I. F., Bryce, M. R., Ahn, J. H., Pearson, C. \& Petty, M. C. New 2,5-diaryl-1,3,4-oxadiazole-fluorene hybrids as electron transporting materials for blended-layer organic light emitting diodes. J. Mater. Chem. 15, 194-203 (2005).

24 Kamtekar, K. T., Wang, C. S., Bettington, S., Batsanov, A. S., Perepichka, I. F., Bryce, M. R., Ahn, J. H., Rabinal, M. \& Petty, M. C. New electroluminescent bipolar compounds for balanced charge-transport and tuneable colour in organic light emitting diodes: triphenylamine oxadiazole-fluorene triad molecules. J. Mater. Chem. 16, 3823-3835 (2006).

25 Guo, X., Cheng, Y. X., Xie, Z. Y., Geng, Y. H., Wang, L. X., Jing, X. B. \& Wang, F. S. Fluorene-based copolymers containing dinaphtho-s-indacene as new building blocks for high-efficiency and color-stable blue LEDs. Macromol. Rapid. Commun. 30, 816-825 (2009).

26 Wu, F. I., Reddy, D. S., Shu, C. F., Liu, M. S. \& Jen, A. K. Y. Novel oxadiazole-containing polyfluorene with efficient blue electroluminescence. Chem. Mater. 15, 269-274 (2003).

27 Shu, C. F., Dodda, R., Wu, F. I., Liu, M. S. \& Jen, A. K. Y. Highly efficient blue-lightemitting diodes from polyfluorene containing bipolar pendant groups. Macromolecules 36, 6698-6703 (2003).

28 Fang, Y. K., Liu, C. L. \& Chen, W. C. New random copolymers with pendant carbazole donor and 1,3,4-oxadiazole acceptor for high performance memory device applications. J. Mater.Chem. 21, 4778-4786 (2011).

29 Ling, Q. D., Wang, W., Song, Y., Zhu, C. X., Chan, D. S. H., Kang, E. T. \& Neoh, K. G. Bistable electrical switching and memory effects in a thin film of copolymer containing electron donor-acceptor moieties and europium complexes. J. Phys. Chem. B. 110, 23995-24001 (2006).

30 Choi, S., Hong, S. H., Cho, S. H., Park, S., Park, S. M., Kim, O. \& Ree, M. High-performance programmable memory devices based on hyperbranched copper phthalocyanine polymer thin films. Adv. Mater. 20, 1766-1771 (2008).

31 Möller, S., Perlov, C., Jackson, W., Taussig, C. \& Forrest, S. R. A polymer/semiconductor write-once read-many-times memory. Nature 426, 166-169 (2003).

32 Mukherjee, B. \& Pal, A. J. Write-once-read-many-times (WORM) memory applications in a monolayer of donor/acceptor supramolecule. Chem. Mater. 19, 1382-1387 (2007).

33 Liu, G., Ling, Q. D., Teo, E. Y. H., Zhu, C. X., Chan, D. S. H., Neoh, K. G. \& Kang, E. T. Electrical conductance tuning and bistable switching in poly(N-vinylcarbazole)-carbon nanotube composite films. Acs Nano 3, 1929-1937 (2009).

34 Kim, D. M., Park, S., Lee, T. J., Hahm, S. G., Kim, K., Kim, J. C., Kwon, W. \& Ree, M. Programmable permanent data storage characteristics of nanoscale thin films of a thermally stable aromatic polyimide. Langmuir 25, 11713-11719 (2009).

35 Möller, S., Forrest, S. R., Perlov, C., Jackson, W. \& Taussig, C. Electrochromic conductive polymer fuses for hybrid organic/inorganic semiconductor memories. J. Appl. Phys. 94, 7811-7819 (2003).

36 Smith, S. \& Forrest, S. R. A low switching voltage organic-on-inorganic heterojunction memory element utilizing a conductive polymer fuse on a doped silicon substrate. Appl. Phys. Lett. 84, 5019-5021 (2004).

$37 \mathrm{Xu}, \mathrm{X}$., Register, R. A. \& Forrest, S. R. Mechanisms for current-induced conductivity changes in a conducting polymer. Appl. Phys. Lett. 89, 1421091 (2006).

38 Li, L., Ling, Q. D. Z Zhu, C. X., Chan, D. S. H., Kang, E. T. \& Neoh, K. G. Bilayer memory device based on a conjugated copolymer and a carbon nanotube/polyaniline composite. J. Electrochem. Soc. 155, H2O5-H2O9 (2008).

39 Wang, B. C., Liao, H. R., Yeh, H. C., Wu, W. C. \& Chen, C. T. Theoretical investigation of stokes shift of 3,4-diaryl-substituted maleimide fluorophores. J. Lumin. 113, 321-328 (2005).

40 Mann, C. K. \& Barnes, K. K. Electrochemical Reactions in Nonaqueous Systems (Marcel Dekker, New York, 1990).

41 Pei, J., Ni, J., Zhou, X., Cao, X. \& Lai, Y. Regioregular head-to-tail oligothiophene-functionalized 9,9'-spirobifluorene derivatives. 2. NMR characterization, thermal behaviors, and electrochemical properties. J. Org. Chem. 67, 8104-8113 (2002). 
42 Kumaresan, D., Thummel, R. P., Bura, T., Ulrich, G. \& Ziessel, R. Color tuning in new metal-free organic sensitizers (bodipys) for dye-sensitized solar cells. Chem. Eur. J. 15, 6335-6339 (2009).

43 Chen, Z. K., Huang, W., Wang, L. H., Kang, E. T., Chen, B. J., Lee, C. S. \& Lee, S. T. A family of electroluminescent silyl-substituted poly(p-phenylenevinylene)s: synthesis, characterization, and structure-property relationships. Macromolecules 33, 90159025 (2000)

44 Yang, C. \& Jenekhe, A. S. Conjugated aromatic polyimines. 2. synthesis, structure, and properties of new aromatic polyazomethines. Macromolecules 28, 1180-1196 (1995).
45 losip, M. D., Destri, S., Pasini, M., Porzioc, W., Pernstich, K. P. \& Batlogg, B. New dithieno[3,2-b:2',3'-d]thiophene oligomers as promising materials for organic field-effect transistor applications. Synth. Met. 146, 251-257 (2004).

46 Frommer, J. E. \& Chance, R. R. in Encylopedia of Polymer Science and Engineering (eds Grayson, M. \& Kroschwitz, J.) 2nd edn, Vol. 5, 462-507 (John Wiley \& Sons, Inc., New York, USA, 1986)

47 Sun, Y. M., Liu, Y. Q. \& Zhu, D. B. Advances in organic field-effect transistors. J. Mater. Chem. 15, 53-65 (2005).

48 Facchetti, A. Semiconductors for organic transistors. Mater. Today. 10, 28-37 (2007). 